\title{
Development of Ultra-High Density Silicon Nanowire Arrays for Electronics Applications
}

\author{
Dunwei Wang ${ }^{1}(\varangle)$, Bonnie A. Sheriff ${ }^{2}$, Michael McAlpine ${ }^{2}$, and James R. Heath ${ }^{2}(\varangle)$ \\ ${ }^{1}$ Department of Chemistry, Boston College, 140 Commonwealth Ave, Chestnut Hill, MA 02467, USA \\ ${ }^{2}$ Division of Chemistry and Chemical Engineering and the Kavli Nanoscience Center, Caltech, 127-72, Pasadena, CA 91125, USA \\ Received: 28 April 2008/ Revised: 17 May 2008/Accepted: 18 May 2008 \\ CTsinghua Press and Springer-Verlag 2008
}

\begin{abstract}
This article reviews our recent progress on ultra-high density nanowires (NWs) array-based electronics. The superlattice nanowire pattern transfer (SNAP) method is utilized to produce aligned, ultra-high density Si NW arrays. We first cover processing and materials issues related to achieving bulk-like conductivity characteristics from 10-20 nm wide Si NWs. We then discuss Si NW-based field-effect transistors (FETs). These NWs \& NW FETs provide terrific building blocks for various electronic circuits with applications to memory, energy conversion, fundamental physics, logic, and others. We focus our discussion on complementary symmetry NW logic circuitry, since that provides the most demanding metrics for guiding nanofabrication. Issues such as controlling the density and spatial distribution of both p- and n-type dopants within NW arrays are discussed, as are general methods for achieving Ohmic contacts to both p- and n-type NWs. These various materials and nanofabrication advances are brought together to demonstrate energy efficient, complementary symmetry NW logic circuits.
\end{abstract}

\section{KEYWORDS}

Ultra-high density nanowire, superlattice nanowire pattern transfer, logic circuit

\section{Introduction}

Nanowires (NWs) are a new class of materials that are often classified as structures with a width that can range from a few to tens of nanometers, and with a length that exceeds the width by at least two orders of magnitude [1-3]. NWs provide alternatives to structures that can be fabricated using lithographic top-down patterning, and are viewed as potential materials for enabling the continued size scaling of electronic devices [4]. In addition, the ability to fabricate high quality semiconductor or metallic NWs opens up a host of unique physical properties for investigation and exploitation. These include plasmonic effects [5], quantum confinement effects [6], and phononic effects [7]. In addition, the intrinsically large surface-to-volume ratio characteristic of all NWs provides new scientific opportunities as well $[8,9]$. These various effects and their associated applications can all be influenced via control over NW dimensions, crystallographic orientation, stoichiometry, surface passivation, doping, etc.

The bulk of the NW literature is centered around materials grown NWs, which fall into two classes. Many types of metal and semimetal NWs can be prepared by electrochemically depositing

Address correspondence to Dunwei Wang, dunwei.wang@bc.edu; James R. Heath, heath@caltech. edu

\section{贯 Springer}


the NW materials into the vertically aligned pores of an anodically etched alumina film [1]. Most semiconductor NWs [2] are produced using the vapor-liquid-solid (VLS) growth method that has been generalized and expanded to a host of semiconductor materials by Lieber's group [10]. VLS NWs require a metal nanoparticle to seed NW growth, and NWs are often prepared as a powder or as a surfacesupported film. While some parameters, such as the concentration of dopants or the NW length, are difficult to control, novel core-shell [11, 12] or branched [13] NW materials, can be prepared. Both the alumina templated and the VLS-synthesized NWs can be prepared with an alternating and controllable stoichiometry along the long axis of the NW [10]. Most of these NWs are investigated for their electronic (or optoelectronic) properties [14]. As a general rule, these NWs need to be organized into the desired device setting, and that still remains a challenge, although significant progress has been made [15-17] .

This article summarizes our work towards harnessing a third class of NWs for electronics applications. Our NWs are not produced by materials growth methods, but instead are patterned using superlattice nanowire pattern transfer, or SNAP. SNAP can be readily harnessed towards the production of metal, insulator, semiconductor, or superconductor NWs, and so is a very general method. It also avoids the difficulties of translating nanowires powders or dispersions into ordered arrangements, since SNAP NWs are intrinsically prepared as a long, aligned NW array with $\sim 1 \mathrm{~nm}$ control obtainable over the NW width and the pitch of the array of NWs. A key advance that we review here involves the development of methods for achieving control over the NW doping levels and the spatial distribution of those dopants, all without damaging the NWs. Such control directly leads to high performance NWs for many fundamental and applied tasks, including field-effect transistor (FET) based logic. An energy efficient approach to FET logic is that of complementary symmetry (CS) logic. CS logic requires a large number of transistors; each p-FET, for example, is balanced by an n-FET within the circuit. The simplest CS logic circuit is an inverter, or NOT gate, which contains a both a
p-FET and an n-FET. While this adds significant fabrication complexity, it also lends significant energy efficiency to CS logic circuits, since the source voltage is always isolated from ground by at least one off-state FET. Patterned and controllable doping procedures make it possible to produce CS logic devices within a single SNAP NW array. CS logic also requires a general solution for Ohmic contacts to both p- and n-FETs. To this end, we discuss a novel patterning technique for forming single crystalline two-dimensional structures within a SNAP NW array that permits the establishment of good and reliable electrical contacts to all types of NWs. In addition to CS logic, this method also opens up opportunities in the areas of quasi-1-D superconducting NWs and high performance thermoelectric NW materials [18, 19]. For CS logic, these advances are all integrated together to produce reasonably complex logic circuits in high yield, including an XOR gate that involves 6 p-type field effect transistor (FETs) and $6 \mathrm{n}$-FETs.

This review is arranged as follows. We first review the SNAP method, and then we summarize our efforts to establish the appropriate doping and nanofabrication methodologies to achieve high quality and controllable Si NW conductivity characteristics. We then discuss work towards achieving high performance FETs from our ultra-high density NW arrays [20]. Toward the end of this section, we present our explorations of new applications, such as nanowire-based sensors, that are enabled by the advances in FET optimizations. We then discuss spatially controlled doping for the production of CS logic [21]. Much of this effort has centered around trying to identify robust and general methods for establishing Ohmic contacts to both p- and n-type Si NWs [22]. While the performance of CS inverters largely provides the metrics for this work, we also discuss our extension of this work to produce more complex CS logic structures, including staged logic that requires full signal restoration of the output voltages [23].

\section{Superlattice nanowire pattern transfer method}

Superlattice nanowire pattern transfer (SNAP) 
translates the atomic control achievable over the individual layer thicknesses within an molecular beam epitaxy (MBE)-grown GaAs / $\mathrm{Al}_{x} \mathrm{Ga}_{(1-x)} \mathrm{As}$ superlattice into a similar level of control over NW width and spacing. It can be utilized to produce highly aligned, high-aspect ratio arrays of metal, insulator, or semiconductor NWs. The only major requirement is that a high quality thin film, from the NWs are to be patterned, must first be obtained. Arrays containing up to 1400 metal or Si NWs have been prepared. Each NW can be as long as a few millimeters. Semiconductor and metal NW widths as small as $7 \mathrm{~nm}$ have been demonstrated, respectively. The NW pitch can be controlled with equal precision, and full-pitch values as small as $13 \mathrm{~nm}$ have been demonstrated.

A brief description of the SNAP method for producing Si NW arrays is shown in Fig. 1, and described here. All steps are carried out within Class 1000 or Class 100 clean room environments. For different NW types, the silicon-on-insulator
(SOI) substrate is replaced with a different thin film. A superlattice consisting of up to 2800 layers (800 layers is typical) of alternating $\mathrm{GaAs}$ and $\mathrm{Al}_{x} \mathrm{Ga}_{(1-x)} \mathrm{As}$ thin films was custom-ordered (IQE, Ltd. Cardiff, $\mathrm{UK})$. A thin ( $2 \mathrm{~mm}$ wide) strip is cleaved from the wafer, and this trip is, in turn, diced into $2-3 \mathrm{~mm}$ long pieces. If the cleaved edges of these pieces do not appear perfectly flat by optical microscopy investigation, they should be discarded. Each piece is immersed in $\mathrm{NH}_{3} / \mathrm{H}_{2} \mathrm{O}_{2} / \mathrm{H}_{2} \mathrm{O}(1: 20: 750 \mathrm{v} / \mathrm{v})$ for a few seconds to selectively etch the $\mathrm{Al}_{x} \mathrm{Ga}_{(1-x)}$ As regions (Fig.1(b)). The resulting edge of the superlattice consists of 50-100 $\mathrm{nm}$ high GaAs ridges separated by $\mathrm{Al}_{x} \mathrm{Ga}_{(1-x)}$ As recessions. Pt metal is deposited using e-beam evaporation onto the edge of the $\mathrm{GaAs}$ ridges (Fig. 1(c)), with the edge of the superlattice held at a $45^{\circ}$ angle to the incident flux of Pt atoms. The Ptcoated superlattice edge is then brought into contact with the doped Si epilayer of the SOI substrate (Fig. 1(d)). Just prior to this step, the epilayer is

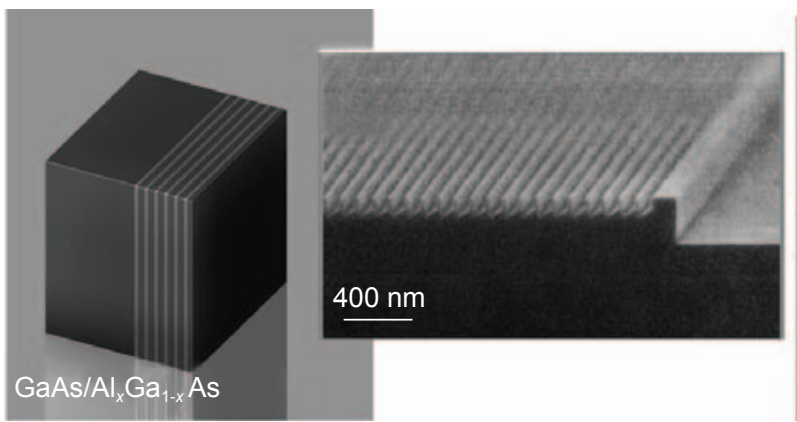

(a)

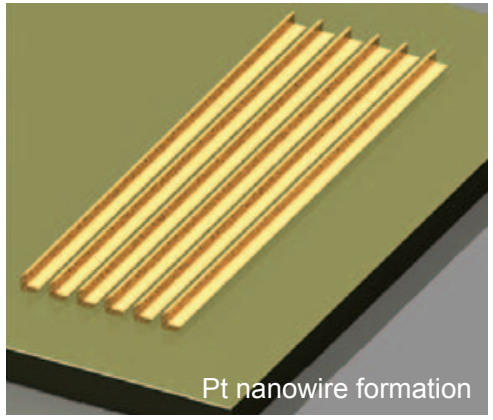

(e)

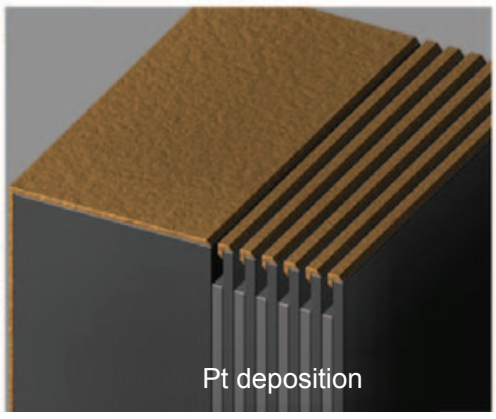

(c)

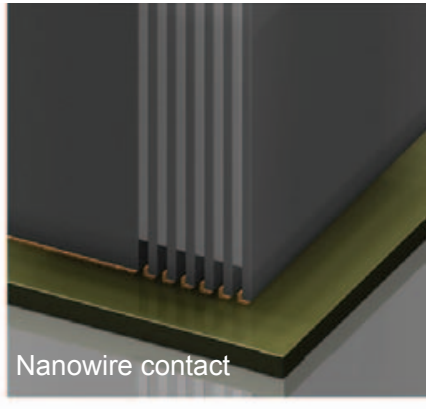

(d)

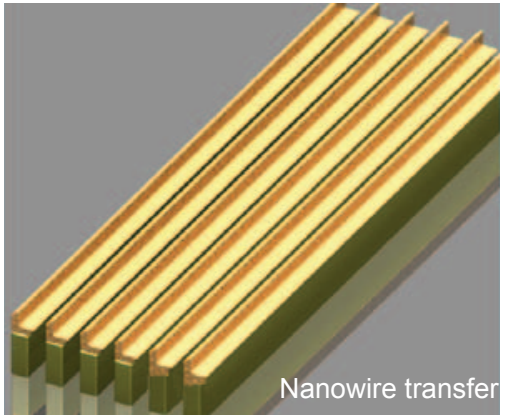

(f)

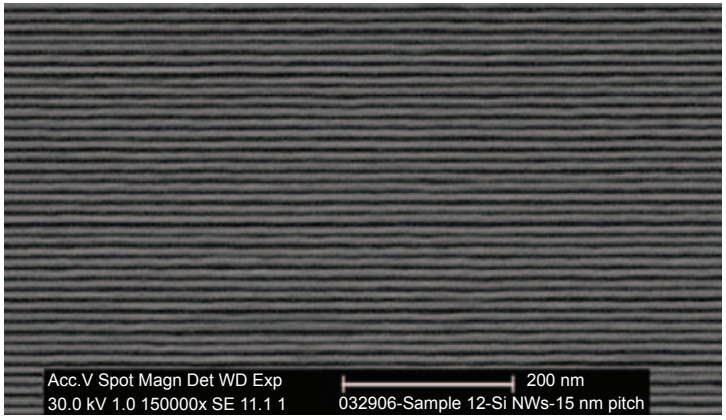

(g)

Figure 1 The SNAP process, shown here for making Si NWs: (a) A small piece of wafer containing a GaAs/Al $\mathrm{Ga}_{1-x}$ As superlattice is cleaved, and the exposed superlattice edge is differentially wet etched; (b) to produce a series of grooves and ridges, shown here is an SEM image; (c) Metal (typically Pt) is evaporated on to that edge at an angle using a well-colliminated electron-beam deposition system; (d) The metalcoated edge of the superlattice chip is dropped onto a PMMA/epoxy $(20 \mathrm{~nm})$ coated SOI wafer so that the metal-coated superlattice ridges are in contact with the silicon epilayers; (e) The superlattice chip is removed using a wet etch, leaving an array of metal NWs behind; ( $f$ ) These nanowires serve as a mask for a directional reactive ion etching; (g) An SEM micrograph of a section of SNAP Si NW array, patterned with an NW width of $7 \mathrm{~nm}$ wide and at a pitch of $13 \mathrm{~nm}$. The scale bar in this micrograph is $200 \mathrm{~nm}$

\section{(1)}

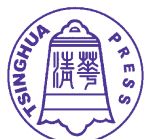

黛 Springer 
covered with a spun-coated thin film (8000 rpm, $30 \mathrm{~s})$ of uncured PMMA/epoxy $(1: 50 \mathrm{w} / \mathrm{w})$. The superlattice/epoxy/SOI assembly is dried on a hot plate $\left(85^{\circ} \mathrm{C}, 30 \mathrm{~min}\right)$ and the superlattice template is then released by a selective etch in $\mathrm{H}_{3} \mathrm{PO}_{4} / \mathrm{H}_{2} \mathrm{O}_{2} / \mathrm{H}_{2} \mathrm{O}$ $(5: 1: 50 \mathrm{v} / \mathrm{v})$ solution, leaving a highly-aligned array of $400 \mathrm{Pt}$ NWs on the surface of the SOI substrate (Fig. 1(e)). These Pt NWs serve as masks for a reactive ion etch (RIE) process to produce aligned, single crystal Si NWs (Fig. 1(e)). The Pt NWs are removed using aqua regia to produce an array Si NWs (Fig. 1(f)) with a width and pitch that is defined by the initial $\mathrm{GaAs} / \mathrm{Al}_{x} \mathrm{Ga}_{(1-x)}$ As superlattice structure, and with a doping and crystal orientation that is inherited from the Si epilayer of the SOI substrate.

After $\mathrm{Si} \mathrm{NW}$ formation, the substrate is cleaned in Piranha $\left(\mathrm{H}_{2} \mathrm{SO}_{4}: \mathrm{H}_{2} \mathrm{O}_{2}=4: 1\right)$ and ALEG (Mallinckrodt Baker, Phillipsburg, NJ) solution to remove contaminants introduced during processing. It is finally treated with an $\mathrm{O}_{2}$ plasma $(20 \mathrm{~W}, 15 \mathrm{~m}$ - Torr, 2 $\mathrm{min})$ to complete the cleaning process.

\section{Achieving bulk-like conductivity charac- teristics from Si NWs}

The ultimate test of the quality of an NW is based upon its electronic properties. For SNAP NWs, those electronic characteristics are inherited from the substrate thin film from which the NWs are made, but are often degraded by the various processing steps of the SNAP method. Furthermore, the highsurface-to-volume ratio of the NWs can also have a detrimental influence. In our initial report of the SNAP method [24], the electrical characteristics of poly-Si wires were measured to be significantly inferior to those of the starting thin films. Thus, we investigated the various parameters that could degrade these electrical characteristics.

Results for a number of SNAP NW arrays prepared using various doping methods and from different starting silicon-on-insulator (SOI) substrates are presented as histograms in Fig. 2 [25]. Several materials and device processing steps can degrade the electrical characteristics of SNAP NWs, but the manner in which the starting substrate is doped is perhaps the most influential such parameter. Ion implantation doping, which is the industry standard, can be harnessed to produce excellent conducting SNAP or electron-beam lithography (EBL) defined Si NWs, as long as the NW widths are $>40-50 \mathrm{~nm}$. However, ion implantation apparently leaves defects, and for thinner NWs, those defects can reduce the conductivity by $10^{3}$ or more (green histogram, Fig. 2). Diffusion-based doping (using below-described spin-on-dopants) is a gentler process. In addition, industry standard 8" SOI wafers are superior to 4" SOI wafers for Si(100) epilayers, and bonded Si

$\langle 111\rangle$ films perform even better. Those $\langle 111\rangle$ bonded films are generally custom-made, and so are not particularly useful for the batch fabrication of devices. Nevertheless, their excellent electrical characteristics prompted us to measure charge carrier mobilities for very thin $(10-20 \mathrm{~nm})\langle 111\rangle$ bonded films. When the native oxide is removed from the top surface of those films and they are methylated (which passivates every atop $\mathrm{Si}$ atom on an unreconstructed Si(111) surface with a methyl group $[26,27])$ bulklike mobilities are observed [28]. For the smoothest of those films, surface scattering of charge carries

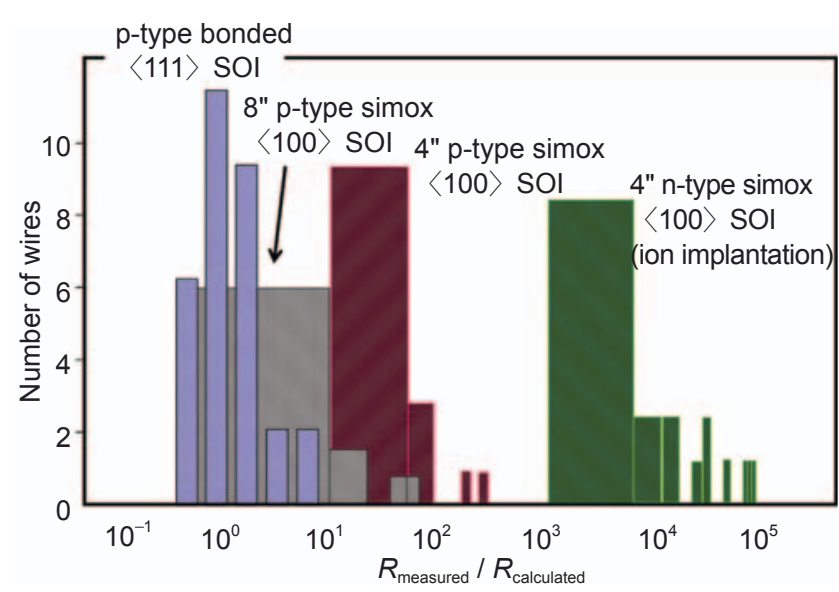

Figure 2 Histograms that chart the influence of device processing and substrate choice on the conductivity of $12-n m$ wide SNAP Si NWs. The figure of merit, $R_{\text {measured }} / R_{\text {calculated, }}$ is based upon the calculated resistivity of the NW assuming it exhibited bulk-like conductivity characteristics (measured for all starting substrates). Ion implantation (green histogram) severely degrades the electronic properties of the $\mathrm{NWs}$ - an effect that is only noticeable for NW widths $<50 \mathrm{~nm}$. For all other histograms, the substrates (and thus the NWs) were doped using thermal diffusion of spin-on-dopants. 8" silicon-on-insulator wafers (the industry standard) are clearly superior to the 4" wafers, and $\langle 111\rangle$ bonded Si wafers (not a standard material) apparently exhibit the best performance, although these particular NWs were 15 $\mathrm{nm}$ wide 
does not appear to play an important role, even at relatively low doping.

\section{Silicon nanowire field effect transistors}

For VLS-grown Si NWs, field effect transistors (FETs) with high mobilities and low subthreshold swings have been reported by the Lieber group [29, 30]. A key towards achieving the excellent conductivity results described above was the use of spin-ondopants (SOD). SODs, which are thermally diffused into the Si epilayers of the SOI substrate prior to the SNAP process, are a class of dopants that have also found uses in devices such as thin film transistors [31, 32]. We optimized SOD for the production of SNAP Si NW FETs.

As-made devices can be characterized by large variations in on-current and on/off switching amplitudes, suggesting the possible presence of defects. Defects can damage performance by acting as scattering centers that reduce carrier mobilities (leading to lower transconductance and lower oncurrent values). They can also serve as charge traps that degrade gating efficiency (poorer on/off ratios and large subthreshold swings) [33]. For p-FETs, the device performance could be significantly improved through a forming gas $\left(5 \% \mathrm{H}_{2}\right.$ in $\left.\mathrm{N}_{2}\right)$ anneal at $475{ }^{\circ} \mathrm{C}$ for $5 \mathrm{~min}$ [34]. For these p-FETs, we find high carrier mobilities, high on-current, excellent on/off ratios, and a low subthreshold swing of hole carriers (Fig. 3). These results indicated that these p-FETs have low numbers of defects, and at least reasonably well passivated surfaces.

The observed improvement of the Si NW FETs following the anneal step can originate from the removal of surface and/or $\mathrm{Si} / \mathrm{SiO}_{2}$ interfacial states, or through the formation of a semiconductor/metal alloy at the $\mathrm{S}$ and $\mathrm{D}$ contacts. It is well known that a forming gas anneal can reduce defects in Si MOSFETs. During annealing, $\mathrm{Si} / \mathrm{SiO}_{2}$ interface dangling bonds are terminated by $\mathrm{H}_{2}$ in forming gas and charge traps diffuse out, directly leading to increased on-current and improved on/off ratios. The effect of producing a metal/semiconductor alloy at the NW/ contact interface would be a decreased Schottky barrier height, manifested as an improved on-current. This is unlikely, however, to significantly reduce offcurrent. We noticed that similar device performance enhancements (i.e., higher on-current and higher on/ off ratios) could be obtained by carrying out the forming gas anneal of the SNAP Si NWs prior to metal contact deposition. Therefore, we suggest that the charge trap removal plays the most important role. The current through our devices (at low $V_{\mathrm{DS}}$ ) varied linearly with NW length, as was previously reported for heavily doped SNAP NWs [35].

SNAP NW FETs were characterized using either the top (deposited) gate electrode, or by employing the supporting Si substrate as a bottom gate. This substrate is separated from the Si epilayer from which the NWs were formed by $150 \mathrm{~nm} \mathrm{SiO}_{2}$. For studies of the SNAP Si NW FETs, local gates with relatively thin $\mathrm{Al}_{2} \mathrm{O}_{3}$ gate dielectrics were deposited on top of $\mathrm{Si}$ NWs. The gate dielectric deposition was carried out in an $\mathrm{O}_{2}$ atmosphere $\left(1 \times 10^{-5}\right.$ Torr $)$ at a rate of $0.1 \AA / \mathrm{s}$, and a total thickness of $100 \AA$ was deposited. The gate electrodes (e-beam evaporated $100 \AA$ Ti and $500 \AA \mathrm{Pt}$ ) overlapped with the S/D contacts. No measurable current leakage was observed between the overlapped $\mathrm{S} / \mathrm{D}$ and gate electrodes.

In comparison with bottom-gated devices, the off-current of top-gated devices is greatly decreased, increasing the on/off ratios by more than one order of magnitude. This originates not only from an improved gating efficiency through the thin $\mathrm{Al}_{2} \mathrm{O}_{3}$ dielectric, but also from the diffusion-based SOD doping process. The distribution of dopants through the thickness of the NWs follows the 1-D diffusion model [33-35]. Therefore, most carriers reside near the top surface of the NWs, making a gate modulation from the top more effective. Once the device has a top gate, the bottom gate modulation is weak compared with the top gate. Using the $I_{\mathrm{DS}}-V_{\mathrm{GS}}$ plot at different $V_{\mathrm{DS}}$, we extracted the hole mobilities $\sim 100 \mathrm{~cm}^{2} /(\mathrm{V} \cdot \mathrm{s})$ from the square law, comparable to bulk $\mathrm{Si}$ values and those reported on Si NWs, as well [20, 29]. For many reported high-performance NW or nanotube (NT) FETs [36, 37], the gate electrode surrounds the sides and the top of the NW or NT, forming a 'multigate' structure. Such a gate can improve the performance of the FETs, but it is impractical for very narrow pitch NW arrays, since the deposited gate materials do 

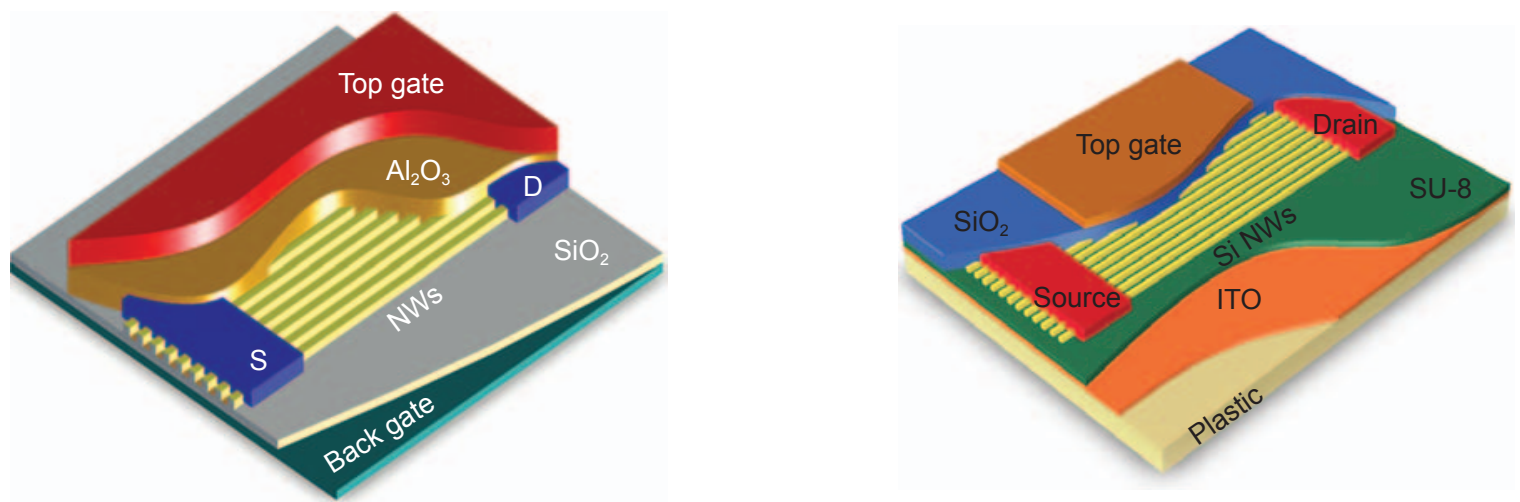

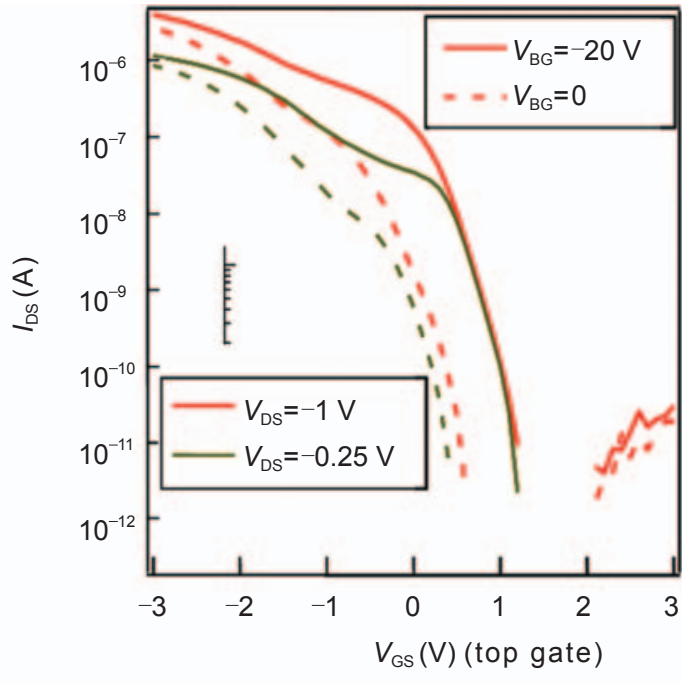

(a)

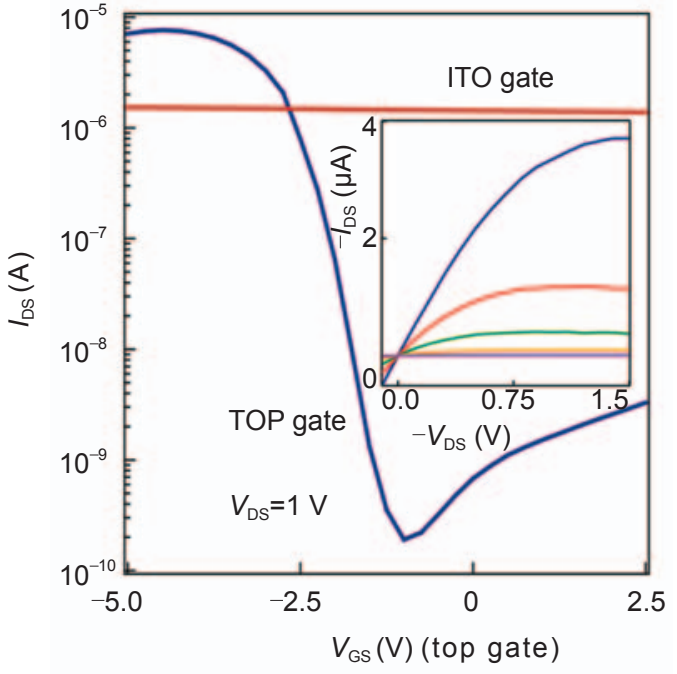

(b)

Figure 3 Si NW p-FETs, fabricated from SNAP NW arrays, exhibit excellent performance on various substrates. At top are schematic drawing of the active area of a Si NW FET fabricated from and supported by an SOI wafer (a) and after transfer to a plastic substrate (b). The electrodes are labeled. The graphs reflect the drain current $\left(I_{D S}\right)$ vs top-gate voltages (VGS). For the SOI device, responses are shown at different bottom gate potentials $\left(V_{\mathrm{BG}}\right)$. For the plastic device, the response to the back gate (red trace) is shown. For either device, once the top-gate has been deposited, bottom gate modulation is limited and the device is dominated by the top gate. The Si NW-on plastic FET contains a larger NW array than does the Si-wafer supported FET, and so the current magnitudes through this device are larger. However, the on/off ratios for both devices are similar. For the plastic device, the inset plot shows $I_{D S}$ VS $V_{D S}$ curves, with the blue, red, green, orange, purple and grey (overlapped by purple) curves corresponding to $V G S=-5,-4,-3,-2,-1$, and $0 \mathrm{~V}$, respectively, applied to the top-gate electrode

not penetrate into the few nanometers wide spaces between the NWs.

NW FETs have a number of applications aside from logic. For example, we have reported on the use of these NWs as highly sensitive biomolecular sensors, detecting as little as 200 attomol/L concentrations of specific DNA oligomers in $0.15 \mathrm{~mol} / \mathrm{L}$ electrolyte solutions [9]. This sensitivity, which is far greater than the sensitivity observed for NW protein sensors in $0.15 \mathrm{~mol} / \mathrm{L}$ electrolyte, arises because the DNA hybridization events occur at top surface of the NWswhere most of the charge carriers reside. We have also demonstrated that the performance of these NW FETs (and NW chemical sensors) can be maintained even after the NWs are transferred onto biocompatible plastic substrates (Fig. 3(b)) [38], similar to what has been observed for VLS NW FETs that are assembled onto plastic substrates [39].

\section{Toward power efficient, complementary symmetry NW logic}

For increasingly dense circuit integration, minimization of static power consumption is critical [40]. With respect to power consumption, logic architectures vary widely. A p-FET, for instance, can be connected 
to a resistor for signal inversion to produce a NOT gate. FET/ resistor logic, however, is energy inefficient - the resistor is dissipative, and the accompanying heat load limits the number of devices that can be packed per unit area. Moreover, such designs do not yield full signal restoration, making it impossible to cascade logic signals through multiple stages - an obvious prerequisite for virtually any type of integrated logic [40]. By replacing the resistor in the above design with an n-FET, one can easily block the direct current pathway from power supply to ground whether the input is high or low, hence greatly reduce the static power consumption. In addition, such a CS design has the potential to exhibit gain and fully restore the output logic voltage level to the input level. Gain implies that the output changes faster than the input. Depending on the quality of the FETs employed, CS logic circuit density can be boosted by orders of magnitude relative to FET/ resistor logic, even though CS logic introduces additional fabrication complexity. This design concept has motivated researchers to fabricate complementary NW devices, including p-type Si/ n-type Si or GaN heterojunctions for diode logic [41, 42], or carbon nanotube (CNT)-based inverters [43, 44]. Nevertheless these systems are limited by both the stochastic chemical nature of the NW formation and doping and by the very challenging issues involving the assembly of devices into even the most basic circuits [41, 43, 45, 46].

CS logic is relatively straightforward using SNAP NW arrays - largely because these NWs inherit their doping profiles from the Si epilayers from which they are formed. Therefore, we can achieve CS doping within a single NW array by predefining the doping patterns within the Si epilayers substrate. The process is illustrated in Fig. 4. Two key advancements are developed here. First, spin-on-glass (SOG) is utilized as a dopants diffusion barrier to define the doping patterns. Second, Si alignment markers guided the placement of individual $\mathrm{p}$ - and n-doping windows and the SNAP NW array mask. The first step of patterned doping was to identify appropriate conditions for both types of dopants, so that a desired doping level can be produced. We tested eight different SODs and chose BoronA (Filmtronics,
Inc.) for p-type and Phosphorousilicate (Emulsitone, Inc.) for n-type, as they provided symmetrical doping results under controllable experimental conditions. The doping conditions and associated results are shown in Fig. 4(b), which also serves as a look-up table for doping condition selections. The doping level was calculated through 4-probe resistivity measurements. Then the film was etched layer by layer in the RIE chamber, $<1 \AA$ for each etching step; doping levels were measured after each etching step, and plotted vs the depth as measured by ellipsometer. Detailed procedures can be found in Ref. [21]. Unlike commonly used metal marks, evaporated Si ones do not contaminate the vicinity areas on the SOI

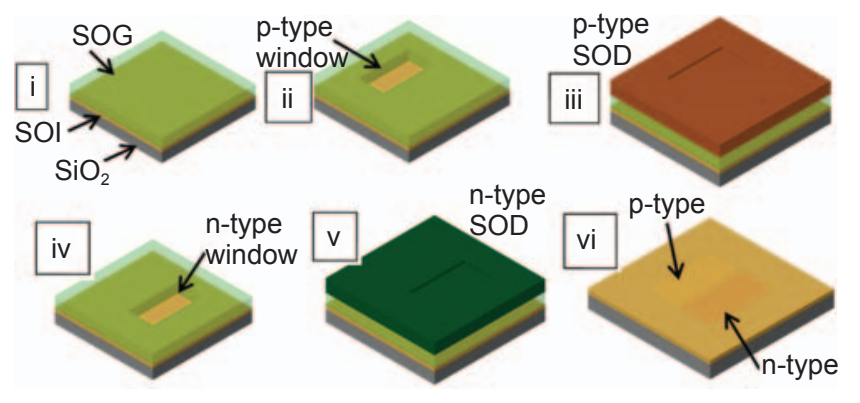

(a)

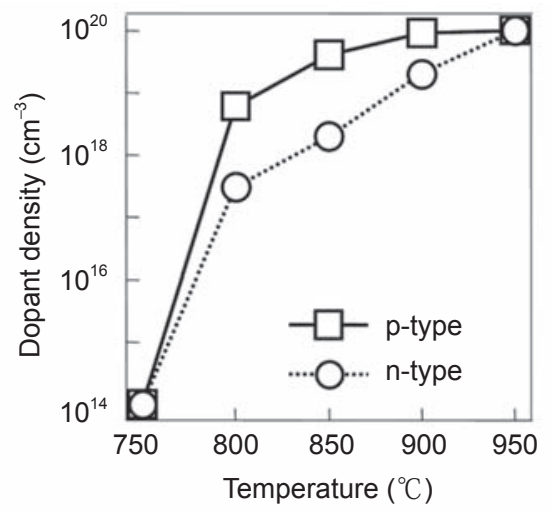

(b)

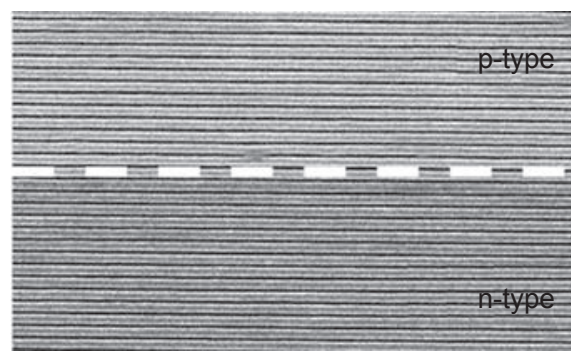

(c)

Figure 4 Process flow (a), thermal parameters (b) for dopant diffusion, and structures (c) of $\mathrm{n}$ - and p-type SNAP Si NWs produced by patterned doping of spin-on-dopants (SODs) 
substrate at high temperatures required for dopants diffusion, therefore was chosen to yield good alignment of the complementary doping patterns. The SNAP process was then conducted on the SOI substrate with predefined doping profiles; care was taken to align the superlattice edges so that the resulting NW arrays contained both p- and n-type NWs. The contrast between these differently doped NWs under electron beam, due to workfunction difference, is manifested in the SEM graph (Fig. 4(c)).

Our first demonstration of CS logic was to take advantage of the patterned doping methods to construct NOT gates by making $\mathrm{p}$ - and n-type NW FETs within the same SNAP array, and then connecting those FETs via metal routing bars after the NWs were already engraved into the SOI substrate [21]. Ti films evaporated in a UHV chamber through electron beam heating were used for $\mathrm{p}$ - and n-FETs, both for the contacts and the gate. Such a simple metallization approach worked well for the first generation devices as the contacts were dominated by surface states on the NWs and gate dielectrics, hence they were insensitive to the workfunctions of contacting metals [21]. Each gate involves one $\mathrm{p}$ - and one n-FET. The p-FETs were optimized as described above. Figure 5(a) shows typical performance of our first generation NOT gates, with reasonable switching performance and a gain of $\sim 5$. However, the output is not fully restored to the input level, and the gain is insufficient for high speed logic applications. The weakest link in these 1st generation NOT gates was the n-FET, which was not optimized.

The optimization of n-FETs for various NWs, including $\mathrm{Si}, \mathrm{Ge}$, and other compound semiconductors, has proven challenging [47-49]. The reasons are related to the relatively low work function, hence high chemical reactivity, of n-type semiconductors. Exposure to ambient air leads to $\mathrm{H}_{2} \mathrm{O}$ and $\mathrm{O}_{2}$ adsorption, which subsequently react with surface atoms, potentially forming mid-gap states [50]. Reliable electrical contacts are difficult to form when the nature of contact is dominated by Fermi level pinning. The challenge is further complicated within the context of CS logic designs, since experimental protocols that work well in optimizing p-FETs can degrade the performance of n-FETs. Our 2nd generation NOT gates involved new concepts for establishing NW contacts and NW-to-NW signal routing lines.

Conventional MOSFET designs utilize metal pads on top of heavily doped semiconductors. These leads to little or no Schottky barrier, and thus Ohmic contacts. It also leaves the active channel regions lightly doped for excellent on/off switching [33]. To emulate part of this approach, we took advantage of the unique doping profile in the SOD-doped SNAP NWs: most dopants reside on the top surface and the dopants concentration decays exponentially through the thickness of the NWs. We formed SNAP NWs that were heavily doped $\left(\sim 10^{19} \mathrm{~cm}^{-3}\right)$. Metal contacts were formed to contact the top surface of the source

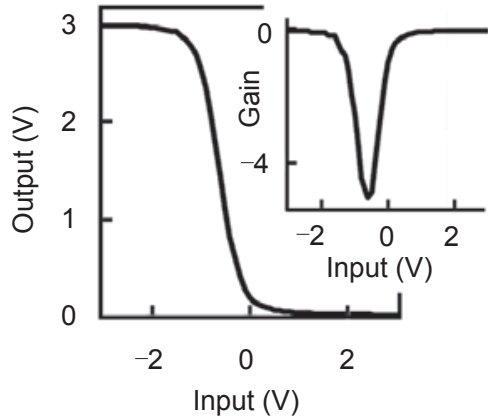

(a)

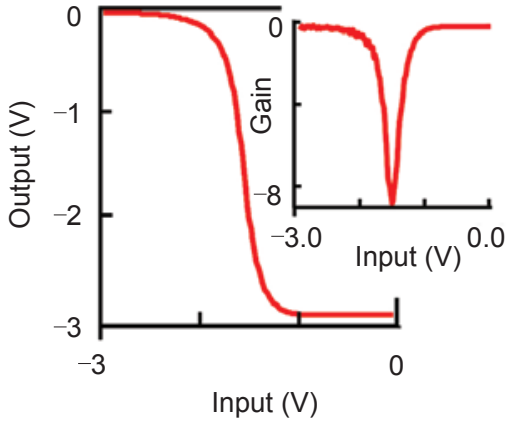

(b)

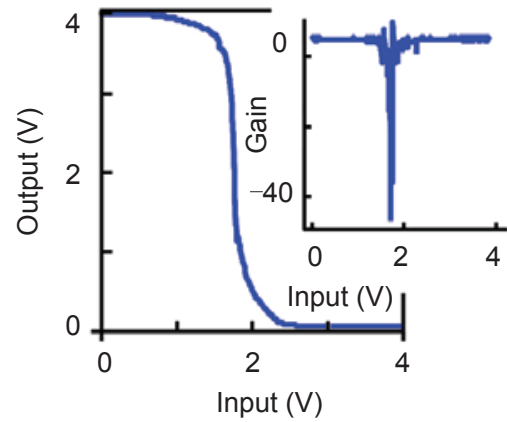

(c)

Figure 5 Several generations of Si NW array-based complementary symmetry inverters (NOT gates). While all working well, the first generation (a) has only the p-FETs optimized and exhibits poor signal restoration and relatively low gain ( 5). (b) This 2nd generation, improved CS inverter has routing bars connecting the p- and n-type NW FETs, as well as large contact pads, that are formed from the same single crystal epilayers from which the NWs are patterned. This greatly improves the quality of the contacts; signal restoration and gain are both significantly improved. (c) Third generation devices were guided by device simulations that suggested the use of different gate materials to better match the $V_{G S} / I_{D S}$ responses of the $\mathrm{p}$ - and $\mathrm{n}$-FETs. Simulations also suggested matching the out currents of the FETs by incorporating different numbers of NWs into the different type FETs. These devices exhibit both full signal restoration and gain approaching 50 
and drain ends of the NWs. The high doping levels ensured Ohmic contacts. The devices were then brought into an RIE chamber and subjected to gentle directional $\mathrm{CF}_{4}$ plasma etching. The contact regions were protected by metal pads and so remained heavily doped, whereas the top layers in the channel regions were removed to yield a much lower channel doping level. This plasma etching process also introduces undesirable surface states, which can be fixed by a $475{ }^{\circ} \mathrm{C}$ forming gas anneal. Taken as a whole, a heterogeneous doping profile that is ideal for FETs operations is created using simple procedures. More importantly, this method works extremely well for both p- and n-FETs. In addition, we note that for complementary symmetry logic, the best matched performance of $n$ - and p-FETs was achieved through the use of Pt gate electrodes on n-FETs and Ti gate electrodes on p-FETs.

The second advance that enabled higher performing NW CS logic gates involved finding patterning methods that could build two-dimensional complexity into the one-dimensional SNAP NW array, i.e., functional components of NWs in one dimension and routing and supporting structures in the other. Accomplishing this, which would be nearly impossible for materials grown NWs, turned out to be fairly straightforward. Electron beam lithography (EBL) was utilized to write patterns into the SNAP NW array after Pt NWs were deposited onto the SOI substrate, but before those Pt NW arrays were translated into Si NW arrays via etching. In this way, the pattern of the Pt SNAP NW array, as well as the EBL pattern, could both be simultaneously translated into the Si epilayer. This method was combined with the above-described technique for establishing ohmic contacts [23]. The result was not only improved NOT-gate performance (Fig. 5(b)) (improved gain and full signal restoration), but also a general method for patterning complex NW-based logic circuits. In addition, the technique of making monolithic structures containing both NWs and submicron sized routing wires and contact pads, is generally applicable to other materials, including metals that serve as models for quasi-1-D superconductors [51], as well as high- $T_{\mathrm{c}}$ superconducting materials [52]. For superconductors in particular, contact issues are always a concern because of proximity effects that can arise when a superconducting material is in contact with a normal state material.

The parameter space for further optimizing CS NW logic circuits is too vast to experimentally explore, and so we turned to computer simulations (in silico methods) for guidance [23, 53]. For those simulations, we established, based upon experimental measurements, look-up table models for both our n- and p-FETs. These tables included current-voltage, capacitance-voltage, and conductance voltage data sets. Once the simulators accurately reproduced the performance of our FETs, then we could rapidly explore extensive parameter space for improving our CS logic circuits. In particular, we focused on finding approaches to better match the performance of our $n$-FETs and p-FETs to each other in terms of both their threshold voltages, and their on and off currents. The simulations suggested that by adjusting the device architecture (using narrower n-FETs to reduce their relative current levels), and using different work function gate electrode materials for the $\mathrm{n}$ - and p-type FETs could dramatically improve NOT gate performance. We then experimentally demonstrated such improvements (Fig. 5(c)). These 3rd generation NOT gates not only exhibited full signal restoration, but also very high gain (approaching 50 for some NOT gates).

\section{Enabled applications}

The capabilities to reliably make ultra-high density NW arrays, and then to both control and organize their electrical properties to meet specific architecture needs, opens up a host of applications. Some compelling examples can be found in our recent publications on binary-tree NW demultiplexing architectures [35], ultra-high density, large-scale molecular electronic memory circuits [54], biomolecular [9] and chemical sensors [38], high performance Si NW thermoelectric materials [18], and superconducting NWs [51]. Relevant to the context of this review article, we focus our discussion on CS logic gates that significant expand upon the concepts described above for NOT gates. Perhaps the most complex 2-input Boolean logic gate is the XOR gate (Fig. 6). For CS logic, this 
gate is composed of 12 FETs ( 6 p- and 6 n-FETs), and requires multiple stages of logic, meaning that signal restoration from one stage is absolutely required to drive the next stage. Thus, the XOR gate provides a very demanding test-bed for illustrating the robustness of the concepts illustrated above for the NOT gates.

XOR gate outputs a logic "1" when one and only one of the inputs is a logic "1". A 2-bit full-adder, for example, involves an XOR gate to sum the two binary numbers and an AND gate to calculate the carry. The AND and OR gates can be approximated by linear functions and, in fact, AND and OR gates can be generated by linear circuit elements using an approach known as resistor or diode logic [55]. In other words, as the input logic values increase from 00 to 01 to 10 to 11 , the output varies from high to

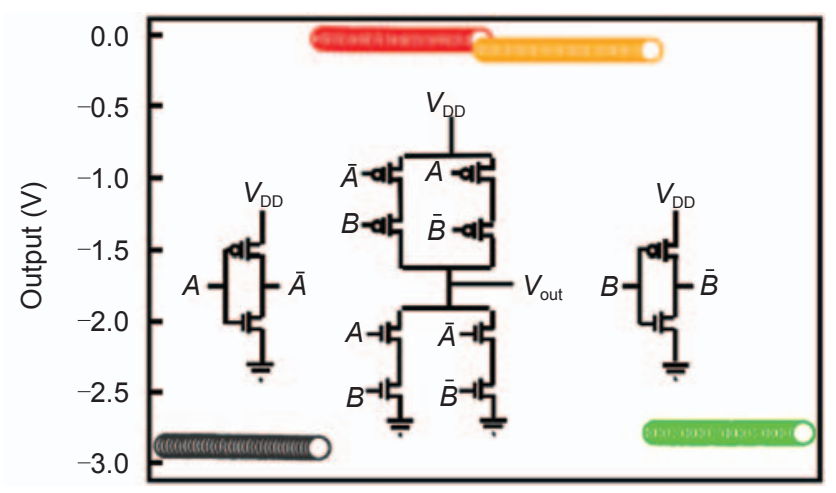

(a)

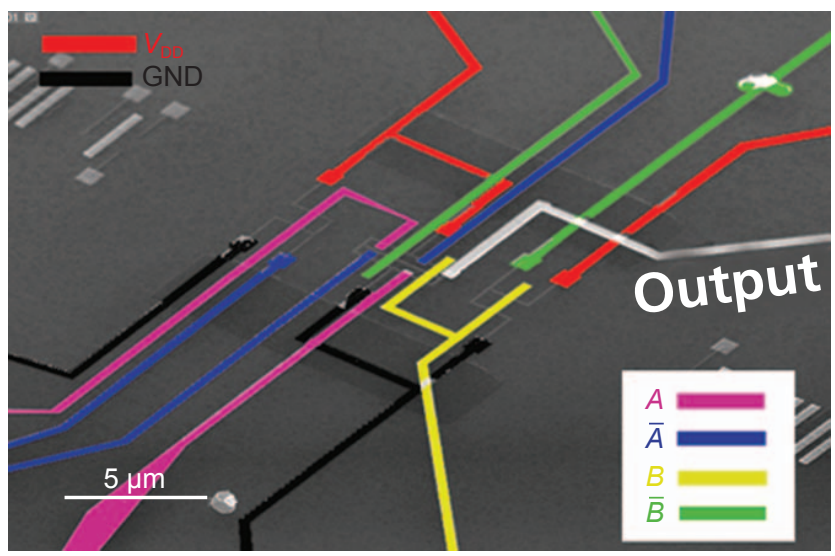

(b)

Figure 6 The most complicated two-input binary logic gate: XOR gates, constructed on monolithically connected Si NWs. The output from left to right in (a) corresponds to input levels of $(1,1),(1,0),(0,1)$, and $(0,0), V_{D D}=-3 \vee$. Equivalent circuits are shown as inset. Electron micrograph is color-coded to distinguish different components for clarity low, or from low to high. For an XOR gate, as the input logic value increases, the output varies from low to high and then back to low-i.e., the XOR gate is intrinsically a nonlinear function. This is the reason that the CS XOR gate requires $6 \mathrm{p}$-FETs and 6 n-FETs. Two inverters are first utilized to create complementary inputs $A$ and $B$ from inputs $A$ and $B$. All four of these logic values are then utilized to drive a circuit of 8 FETs, with each input utilized to drive a p-FET and an n-FET. The detailed equivalent circuit, along with an SEM picture of an XOR gate are shown in Fig. 6. The interconnects between different stages (such inverters to produce $A$ and $B$ ) are not seen in the view field. When all 12 FETs operate consistently, a functional XOR gate is achieved with the appropriate truth table that exhibits full signal restoration.

We tested 15 XOR gates and found 5 of them worked appropriately. If a single NW FET performed poorly within an XOR gate, the XOR gate would fail, and we assume that this is the dominant failure mode. There were 180 FETs tested in the 15 XOR gates, and the $33 \%$ yield in XOR gates implies an approximately 93\% yield in working FETs. It is worth noting that the requirement that the NW FET fall into the operational range of these CS logic gates is a very stringent one. A likely failure mechanism may originate from the poor gate dielectric, which was electron-beam evaporated $\mathrm{Al}_{2} \mathrm{O}_{3}$. If that dielectric were replaced with a more reliable material and reliable deposition method, such as high- $\kappa$ materials deposited by atomic layer deposition (ALD) [56], we would expect not only an improved yield but significantly better CS logic gate performance.

\section{Conclusions}

The SNAP method has been refined to allow for quite sophisticated demonstrates of NW-based memory and logic circuits, with device densities that are well-beyond what can be achieved using alternative techniques. However, we have only begun to explore the applications of the SNAP method towards producing NWs of materials other than $\mathrm{Si}$, and there is surely a rich area of physics and materials science to be explored there. In addition, while the materials 
grown nanowires are difficult to assemble into organized structures, they do provide the opportunity for mixing and matching various types and classes of NWs. Coupling the precision of the SNAP technique with the unique characteristics of materials grown nanowires and nanoparticles also provides interesting avenues for future exploration - with applications that can range from energy harvesting to optoelectronics, and may others.

\section{Acknowledgements}

This research was executed at Caltech and supported by a subcontract from the MITRE Corporation, the MARCO center for Advanced Materials and Devices, and the National Science Foundation (NMF-CCF05204490 and CCF-0541461).

\section{References}

[1] Lieber, C. M. Nanoscale science and technology: building a big future from small things. MRS Bull. 2003, 28, 486491.

[2] Xia, Y. N.; Yang, P. D. Chemistry and physics of nanowires. Adv. Mater. 2003, 15, 351.

[3] Yang, P. D. The chemistry and physics of semiconductor nanowires. MRS Bull. 2005, 30, 85-91.

[4] The international technology roadmap for semiconductors (ITRS): process integration, devices, and structures (Semiconductor Industry Association, San Jose, California, 2005). <http://www.itrs.net/reports.html>

[5] Maier, S. A.; Brongersma, M. L.; Kik, P. G.; Meltzer, S.; Requicha, A. A. G.; Atwater, H. A. Plasmonics-A route to nanoscale optical devices. Adv. Mater. 2001, 13, 1501.

[6] Brus, L. Electronic wave-functions in semiconductor clusters-Experiment and theory. J. Phys. Chem. 1986, 90, 2555-2560.

[7] Caruso, F. Nanoengineering of particle surfaces. Adv. Mater. 2001, 13, 11.

[8] Wang, D. W.; Chang, Y. L.; Wang, Q.; Cao, J.; Farmer, D. B.; Gordon, R.G.; Dai, H. J. Surface chemistry and electrical properties of germanium nanowires. J. Am. Chem. Soc. 2004, 126, 11602-11611.

[9] Bunimovich, Y. L.; Shin, Y. S.; Yeo, W. S.; Amori, M.; Kwong, G.; Heath, J. R. Quantitative real-time measurements of DNA hybridization with alkylated nonoxidized silicon nanowires in electrolyte solution. J. Am. Chem. Soc. 2006, 128, 16323-16331.

[10] Lieber, C. M. Nanoscale science and technology: Building a big future from small things. MRS Bull. 2003, 28, 486491.

[11] Lauhon, L. J.; Gudiksen, M. S.; Wang, C. L.; Lieber, C. M. Epitaxial core-shell and core-multishell nanowire heterostructures. Nature 2002, 420, 57-61.

[12] Gudiksen, M. S.; Lauhon, L. J.; Wang, J.; Smith, D. C.; Lieber, C. M. Growth of nanowire superlattice structures for nanoscale photonics and electronics. Nature 2002, 415, 617-620.

[13] Wang, D.; Qian, F.; Yang, C.; Zhong, Z. H.; Lieber, C. M. Rational growth of branched and hyperbranched nanowire structures. Nano Lett. 2004, 4, 871-874.

[14] Xia, Y. N.; Yang, P. D.; Sun, Y. G.; Wu, Y. Y.; Mayers, B.; Gates, B.; Yin, Y. D.; Kim, F.; Yan, Y. Q. One-dimensional nanostructures: Synthesis, characterization, and applications. Adv. Mater. 2003, 15, 353-389.

[15] Jin, S.; Whang, D. M.; Mcalpine, M. C.; Friedman, R. S.; Wu, Y.; Lieber, C. M. Scalable interconnection and integration of nanowire devices without registration. Nano Lett. 2004, 4, 915-919.

[16] Wang, D. W.; Chang, Y. L.; Liu, Z.; Dai, H. J. Oxidation resistant germanium nanowires: Bulk synthesis, long chain alkanethiol functionalization, and LangmuirBlodgett assembly. J. Am. Chem. Soc. 2005, 127, 1187111875.

[17] Javey, A.; Nam, S.; Friedman, R. S.; Yan, H.; Lieber, C. M. Layer-by-layer assembly of nanowires for threedimensional, multifunctional electronics. Nano Lett. 2007, 7, 773-777.

[18] Boukai, A. I.; Bunimovich, Y.; Tahir-Kheli, J.; Yu, J. -K.; Goddard III, W.A.; Heath, J.R. Silicon nanowires as efficient thermoelectric materials. Nature 2008, 451, 168-171.

[19] Hochbaum, A. I.; Chen, R.; Delgado, R. D.; Liang, W.; Garnett, E. C.; Najarian, M.; Majumdar, A.; Yang, P. Enhanced thermoelectric performance of rough silicon nanowires. Nature 2008, 451, 163-167.

[20] Wang, D. W.; Sheriff, B. A.; Heath, J. R. Silicon p-FETs from ultrahigh density nanowire arrays. Nano Lett. 2006, 6, 1096-1100.

[21] Wang, D. W.; Sheriff, B. A.; Heath, J. R. Complementary symmetry silicon nanowire logic: Power-efficient inverters with gain. Small 2006, 2, 1153-1158. 
[22] Liu, B. Z.; Wang, Y. F.; Dilts, S.; Mayer, T. S.; Mohney, S. E. Silicidation of silicon nanowires by platinum. Nano Lett. 2007, 7, 818-824.

[23] Wang, D. W.; Sheriff, B. A.; Heath, J. R.; Kurtin, J. N. Complementary symmetry nanowire logic circuits: Experimental demonstrations and in silico optimizations. ACS Nano 2008, revised, acceptance pending.

[24] Melosh, N. A.; Boukai, A.; Diana, F.; Gerardot, B.; Badolato, A.; Petroff, P. M.; Heath, J. R. Ultrahigh-density nanowire lattices and circuits. Science 2003, 300, 112115.

[25] Beckman, R. A.; Johnston-Halperin, E.; Melosh, N. A.; Luo, Y.; Green, J. E.; Heath, J. R. Fabrication of conducting Si nanowire arrays. J. Appl. Phys. 2004, 96, 5921-5923.

[26] Yu, H. B.; Webb, L. J.; Ries, R. S.; Solares, S. D.; Goddard, W. A.; Heath, J. R.; Lewis, N. S. Low-temperature STM images of methyl-terminated Si (111) surfaces. J. Phys. Chem. B 2005, 109, 671-674.

[27] Solares, S. D.; Yu, H. B.; Webb, L. J.; Lewis, N. S.; Heath, J. R.; Goddard, W. A. Chlorination-methylation of the hydrogen-terminated silicon (111) surface can induce a stacking fault in the presence of etch pits. J. Am. Chem. Soc. 2006, 128, 3850-3851.

[28] Green, J. E.; Wong, S. J.; Heath, J. R. Hall mobility measurements and chemical stability of ultrathin, methylated Si (111)-on-insulator films. J. Phy. Chem. C 2008, 112, 5185-5189.

[29] Cui, Y.; Zhong, Z. H.; Wang, D. L.; Wang, W. U.; Lieber, C. M. High performance silicon nanowire field effect transistors. Nano Lett. 2003, 3, 149-152.

[30] Mcalpine, M. C.; Friedman, R. S.; Jin, S.; Lin, K. H.; Wang, W. U.; Lieber, C. M. High-performance nanowire electronics and photonics on glass and plastic substrates. Nano Lett. 2003, 3, 1531-1535.

[31] Menard, E.; Nuzzo, R. G.; Rogers, J. A. Bendable single crystal silicon thin film transistors formed by printing on plastic substrates. Appl. Phys. Lett. 2005, 86, 093507.

[32] Zhu, Z. T.; Menard, E.; Hurley, K.; Nuzzo, R. G.; Rogers, J. A. Spin on dopants for high-performance single-crystal silicon transistors on flexible plastic substrates. Appl. Phys. Lett. 2005, 86, 133507.

[33] Sze, S. M. Physics of Semiconductor Devices; Wiley: New York, 1981.

[34] Wolf, S.; Tauber, R. N. Silicon Processing for the VLSI Era, 2nd ed.; Lattice Press: Sunset Beach, CA, 2000.

[35] Beckman, R.; Johnston-Halperin, E.; Luo, Y.; Green, J.
E.; Heath, J. R. Bridging dimensions: Demultiplexing ultrahigh-density nanowire circuits. Science 2005, 310, 465-468.

[36] Javey, A.; Guo, J.; Paulsson, M.; Wang, Q.; Mann, D. Lundstrom, M.; Dai, H. J. High-field quasiballistic transport in short carbon nanotubes. Phys. Rev. Lett. 2004, 92.

[37] Koo, S. M.; Li, Q.; Monica, D. E.; Richter, C. A.; Vogel, E. $M$. Enhanced channel modulation in dual-gated silicon nanowire transistors. Nano Lett. 2005, 5, 2519-2523.

[38] Mcalpine, M. C.; Ahmad, H.; Wang, D.; Heath, J. R. Highly ordered nanowire arrays on plastic substrates for ultrasensitive flexible chemical sensors. Nat. Mater. 2007, 6, 379-384.

[39] Mcalpine, M. C.; Friedman, R. S.; Lieber, C. M. Highperformance nanowire electronics and photonics and nanoscale patterning on flexible plastic substrates. Proc. IEEE 2005, 93, 1357-1363.

[40] Rabaey, J. M.; Chandrakasan, A.; Nikolic, B. Digital Integrated Circuits: A Design Perspective, 2nd ed.; Pearson Education, Inc.: Upper Saddle River, NJ, 2003.

[41] Cui, Y.; Lieber, C. M. Functional nanoscale electronic devices assembled using silicon nanowire building blocks. Science 2001, 291, 851-853.

[42] Huang, Y.; Duan, X. F.; Cui, Y.; Lauhon, L. J.; Kim, K. $\mathrm{H}$.; Lieber, C. M. Logic gates and computation from assembled nanowire building blocks. Science 2001, 294, 1313-1317.

[43] Chen, Z. H.; Appenzeller, J.; Lin, Y. M.; Sippel-Oakley, J.; Rinzler, A. G.; Tang, J. Y.; Wind, S. J.; Solomon, P. M.; Avouris, P. An integrated logic circuit assembled on a single carbon nanotube. Science 2006, 311, 1735.

[44] Javey, A.; Wang, Q.; Ural, A.; Li, Y. M.; Dai, H. J. Carbon nanotube transistor arrays for multistage complementary logic and ring oscillators. Nano Lett. 2002, 2, 929-932.

[45] Chen, Y.; Jung, G. Y.; Ohlberg, D. A. A.; Li, X. M.; Stewart, D. R.; Jeppesen, J. O.; Nielsen, K. A.; Stoddart, J. F.; Williams, R. S. Nanoscale molecular-switch crossbar circuits. Nanotechnology 2003, 14, 462-468.

[46] Chau, R.; Datta, S.; Doczy, M.; Doyle, B.; Jin, J.; Kavalieros, J.; Majumdar, A.; Metz, M.; Radosavljevic, M. Benchmarking nanotechnology for high-performance and low-power logic transistor applications. IEEE T. Nanotechnol. 2005, 4, 153-158.

[47] Zheng, G. F.; Lu, W.; Jin, S.; Lieber, C. M. Synthesis and fabrication of high-performance n-type silicon nanowire transistors. Adv. Mater. 2004, 16, 1890-1893. 
[48] Wang, D. W.; Chang, Y. L.; Wang, Q.; Cao, J.; Farmer, D. B.; Gordon, R. G.; Dai, H. J. Surface chemistry and electrical properties of germanium nanowires. J. Am. Chem. Soc. 2004, 126, 11602-11611.

[49] Duan, X. F.; Niu, C. M.; Sahi, V.; Chen, J.; Parce, J. W.; Empedocles, S.; Goldman, J. L. High-performance thinfilm transistors using semiconductor nanowires and nanoribbons. Nature 2003, 425, 274-278.

[50] Kingston, R. H. Semiconductor Surface Physics University of Pennsylvania Press: Philadelphia, 1957.

[51] Xu, K.; Heath, J. R. Controlled fabrication and electrical properties of long quasi-one-dimensional superconducting nanowire arrays. Nano Lett. 2008, 8, 136-141.

[52] Xu, K.; Heath, J. R. submitted, 2008.

[53] Bindal, A.; Hamedi-Hagh, S. The impact of silicon nanowire technology on the design of single-work-function
CMOS transistors and circuits. Nanotechnology 2006, $17,4340-4351$.

[54] Green, J. E.; Wook Choi, J.; Boukai, A.; Bunimovich, Y.; Johnston-Halperin, E.; Deionno, E.; Luo, Y.; Sheriff, B.A.; Xu, K.; Shik Shin, Y.; Tseng, H. -R.; Stoddart, J. F.; Heath, J. R. A 160-kilobit molecular electronic memory patterned at 1011 bits per square centimetre. Nature 2007, 445, 414-417.

[55] Horowitz, P.; Hill, W. The Art of Electronics. Cambridge: Cambridge University Press, 1989.

[56] Wang, D. W.; Wang, Q.; Javey, A.; Tu, R.; Dai, H. J.; Kim, H.; Mcintyre, P. C.; Krishnamohan, T.; Saraswat, K. C. Germanium nanowire field-effect transistors with $\mathrm{SiO}_{2}$ and high-kappa $\mathrm{HfO}_{2}$ gate dielectrics. Appl. Phys. Lett. 2003, 83, 2432-2434. 